\title{
Cardiff Behavioural Model Analysis using a Two-Tone Stimulus
}

\begin{abstract}
This paper presents a new technique for identifying the mixing structure, model coefficients and therefore model order of the Cardiff behavioral model for phase related nonlinearities. The technique employs a two-tone measurements approach and the Fast Fourier Transform (FFT) to be able to observe the mixing structure above the noise floor of the measurement system. Spectral tone visibility explicitly requires model coefficient inclusion for accurate $(\mathrm{NMSE}<-40 \mathrm{~dB})$ data fitting, which is verified by comparing model fitting of full and truncated model formulations. The identified maximum phase model order from two-tone measurements, for annuli on the Smith Chart, is shown to be accurate for Continuous Wave (CW) measurements.
\end{abstract}

Index Terms-Behavioral model, Two-tone stimulus, device nonlinear models.

\section{INTRODUCTION}

Behavioral models are a necessary tool to transport device data, from a developing technology, into CAD for further design related analysis. The Cardiff Model exploits the property that when multiple stimuli are injected into a multi-port nonlinear system they interact or 'mix'. The Cardiff Model is defined in the frequency domain and when correctly implemented should include all the resulting frequency components. Hence, the Cardiff Model differentiates itself from other popular behavioral modeling formulations, S-parameters, Polyharmonic Distortion (PHD) modeling [1], [2] and X-parameters [3], by not limiting the number of model coefficients that can be extracted; for example, 2 coefficients for S-parameters and 3 for analytical X-parameters and PHD modeling. This approach allows for a global model fit of data, obtained by executing impedance sweeps over an area of the Smith Chart, rather than a local fit about/at each impedance point of the sweep. Previous work in [4] has verified the Cardiff Model coefficient structure so that global models of harmonic sourceand load-pull data can be accurately extracted with attempts at avoiding over-fitting the data.

In this paper, a new technique for determining the correct, and present, phase polynomial coefficients of the Cardiff Model will be presented. This technique utilizes two-tone measurements and the Fast Fourier Transform (FFT) to be able to observe the mixing order and resultant intermodulation (IMD) products and demonstrate that they are directly correlated to the modeling coefficients used in the Cardiff Model. This approach allows for the direct and accurate determination of the maximum number of model phase coefficients needed for two-tone and $\mathrm{CW}$ measurements.

\section{The MEASUREMENT SYSTEM ARCHITECTURE AND PROCEDURE}

The measurement system for this paper utilizes two Arbitrary Waveform Generators (AWG) to enable two-tone generation, and four Vector Signal Analyzers (VSAs) for travelingwave acquisition. Fig. 1 shows a block diagram of the measurement system, which is comprised of the aforementioned AWGs and VSAs and also driver PAs, filters, and a calibrated test-set. The system architecture is fundamentally linked to previous work on Vector Network Analyzer (VNA) based systems [5]. Fig. 1 also depicts the measurement scenario by showing the DUT input driven by a single tone and the DUT output driven by a two-tone stimulus. The chosen DUT is the Wolfspeed $10 \mathrm{~W}$ packaged device, biased at $\mathrm{Vgs}=-2.8 \mathrm{~V}$ and $\mathrm{Vds}=28 \mathrm{~V}$, driven with $\left|a_{11}\right|=24.65 \mathrm{dBm}$.

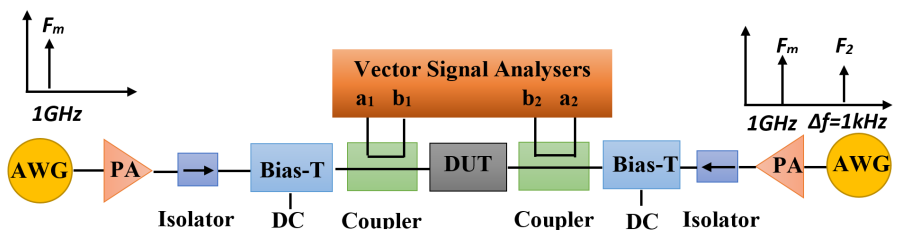

Fig. 1. Block diagram of the measurement system.

The VSAs have an instantaneous $50 \mathrm{MHz}$ bandwidth, which allows for the acquisition of the down converted two tones and IMD products without changing the Local Oscillator (LO) frequency. Due to limits on the calibration coefficients being able to extend over a $50 \mathrm{MHz}$ bandwidth, the main-tone frequency was chosen to be $1 \mathrm{GHz}$ and the tone frequency spacing was limited to $1 \mathrm{kHz}$; very close to the calibrated frequency.

To ensure the data was design relevant, the main tone impedance was located at the DUTs optimum power point on the Smith Chart, using the algorithm in [6]. To be able to investigate the presence of model terms and their relation to the IMD spectra, the $2^{\text {nd }}$ output tone magnitude $\left|a_{21,2}\right|$ was varied from $-6 \mathrm{dBc}$ to $+6 \mathrm{dBc}$ of the main tone signal magnitude, $\left|a_{21,1}\right|$. This will create growing levels of observable distortion in the $b$-wave response and hence a growing model complexity and load modulation coverage of the Smith Chart.

\section{SPECTRAL ANALYSIS OF THE BEHAVIORAL MODEL}

In order to analyze the received, down converted spectra, the selected tones in the receiver bandwidth needed to be calibrated and phase-normalized to the input traveling-wave $\left(a_{11}\right)$ at the main tone frequency $(1 \mathrm{GHz})$. This established 
a relative phase system, key for behavioral modeling and analysis [2]-[4]. The raw, down converted IMD tones lie on a harmonic grid $\left( \pm \mathrm{n}^{*} 1 \mathrm{kHz}\right)$ and were compared to a proposed noise floor of $-85 \mathrm{~dB}$ for selection before calibration. Using the IFFT, the time-domain traveling-wave quantities were then constructed from the selected tones. This allowed for b-wave analysis and load modulation coverage to be observed on the Smith Chart. All noise tones were removed in this process. After identifying the maximum model order for the twotone measurements, selected impedances were used for $\mathrm{CW}$ measurement and model analysis was performed to verify whether the identification holds for the $\mathrm{CW}$ domain.

Fig. 2 (a) depicts the down converted $\left|b_{21 E}\right|$ (the b-envelope about the fundamental tone) spectral plot, for $\left|a_{21,2}\right|=-6 \mathrm{dBc}$, and links the observable tones, above the noise floor, to Cardiff Model coefficients $\left(K_{p h, m, \varphi}\right)$. The 'p' and ' $h$ ' subscripts denote the respective port and harmonic, and the ' $m$ ' and ' $\varphi$ ' subscripts denote the coefficient's related traveling-wave mag and phase exponent respectively (see eq.1). Overlaid, on the model tones is the associated model term contribution (e.g., $\left.K_{21,1,1}\left|a_{21 E}\right|\left(\frac{Q}{P}\right)\right)$ after extracting the model for eq. 1 . The almost exact overlap indicates the accuracy of the model formulation's ability to fit the data.

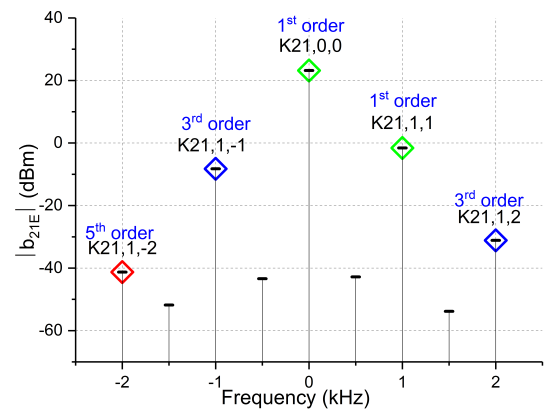

(a) $\left|b_{21 E}\right|$ spectrum showing $5^{\text {th }}$ order coefficients.

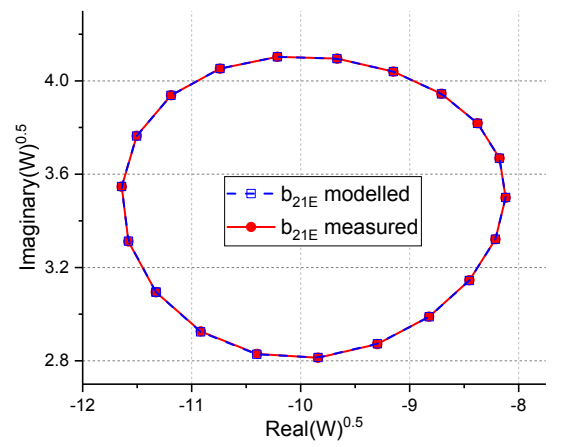

(b) $b_{21 E}$ comparison for the selected coefficients.

Fig. 2. $b_{21 E}$ analysis for $\left|\mathrm{a}_{21,2}\right|=-6 \mathrm{dBc}$.

Equation 1 formulates the required Cardiff Model in this case based on the observations from the spectra in fig. 2 (a) this is a relative phase polynomial only, as each annulus is taken at a fixed $\left|a_{21}\right|$ injection and the relative phase is the only variable. Fig. 2 (b) shows the comparison between $b_{21 E}$ model and measured data, it clearly shows an accurate model fit to the data and verifies the fit of the model terms, in eq. 1, to the spectral tones, in fig. 2 (a).

$$
\begin{aligned}
\frac{b_{21}}{P}= & K_{(21,0,0)}+K_{(21,1,1)}\left|a_{21 E}\right|\left(\frac{Q}{P}\right)+ \\
& K_{(21,1,-1)}\left|a_{21 E}\right|\left(\frac{Q}{P}\right)^{-1}+K_{(21,1,2)}\left|a_{21 E}\right|\left(\frac{Q}{P}\right)^{2} \\
& K_{(21,1,-2)}\left|a_{21 E}\right|\left(\frac{Q}{P}\right)^{-2}
\end{aligned}
$$

Fig. 3 (a) shows the resulting spectrum for $\left|a_{21,2}\right|=0 \mathrm{dBc}$, and indicates that stronger mixing process are present, which requires a commensurate model formulation $\left(9^{\text {th }}\right.$ order $)$ for accurate model extraction. Again, the overlaid extracted model term contributions map directly onto the spectral lines. Fig. 3 (b) shows another good model fit to the measured data.

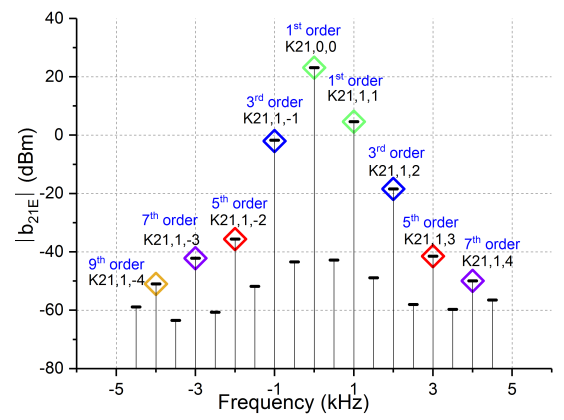

(a) $\left|b_{21 E}\right|$ spectrum showing $9^{\text {th }}$ order coefficients.

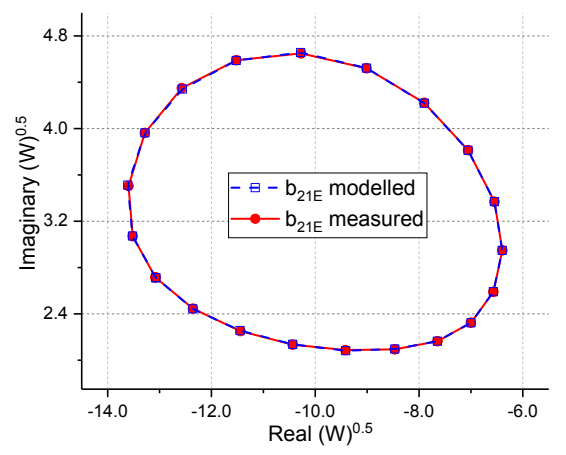

(b) $b_{21 E}$ comparison for the selected coefficients.

Fig. 3. $b_{21 E}$ analysis for $\left|\mathrm{a}_{21,2}\right|=0 \mathrm{dBc}$.

The final measurement, where $\left|a_{21,2}\right|=6 \mathrm{dBc}$ of the main tone, was performed to cover a significant area of the Smith Chart. Fig. 4 shows the resultant $\left|b_{21 E}\right|$ spectrum, from which the Cardiff Model equation was formulated commensurate with the observed model order.

Fig. 5 shows the comparison between model and measurement data for full and truncated models. To demonstrate the inaccuracy of model truncation, the full $11^{\text {th }}$ order Cardiff Model, in (a), has been compared with the 3-term analytical Xparameter model, in (b), and clearly shows a limitation of the analytical X-parameter model for this $\left|a_{21,2}\right|$. However, $\left|a_{21,2}\right|$ 


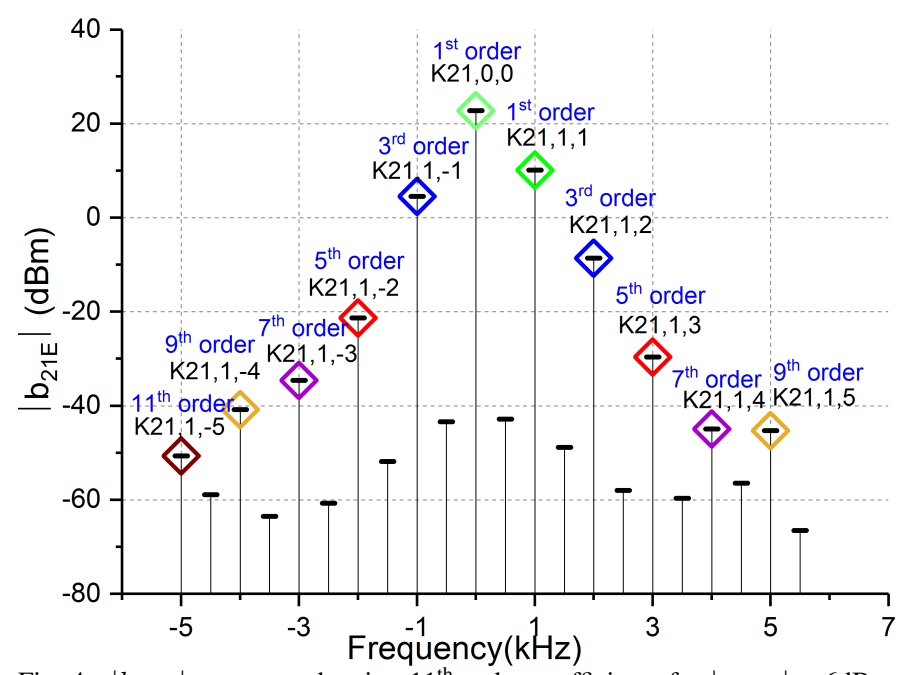

Fig. 4. $\left|b_{21 E}\right|$ spectrum showing $11^{\text {th }}$ order coefficients for $\left|a_{21,2}\right|=6 \mathrm{dBc}$.

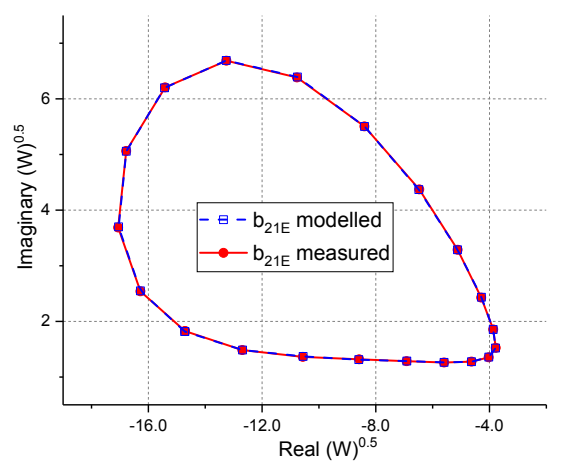

(a) $b_{21 E}$ comparison for 11 coefficients (Cardiff model).

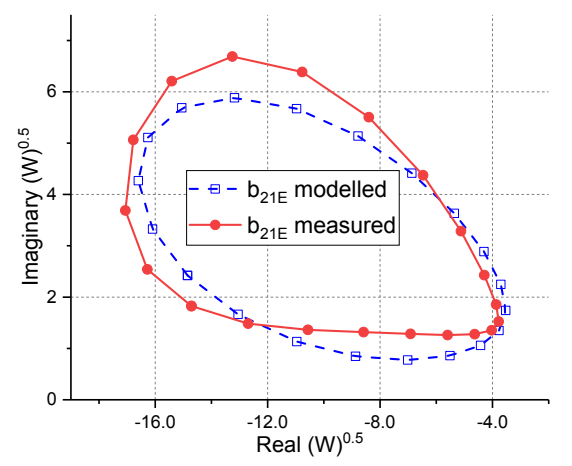

(b) $b_{21 E}$ comparison for 3 coefficients (analytical X-parameter model).

Fig. 5. $b_{21 E}$ analysis for $\left|\mathrm{a}_{21,2}\right|=6 \mathrm{dBc}$.

$=2 \mathrm{dBc}$ is where the analytical X-parameter formulation begins to produce a normalized mean square error (NMSE) $>-40 \mathrm{~dB}$, above $1 \%$ error, below $2 \mathrm{dBc}$ the error is acceptable but not optimal.

Fig. 6 shows all the annuli collected in the measurement process with each annulus being modeled with its corresponding, spectrally-identified, behavioral model equation. Fig. 7 shows the impedance area covered by the load-modulation that occurs from the two-tone measurements. The $-6 \mathrm{dBc}, 0 \mathrm{dBc}$, and $6 \mathrm{dBc}$ annuli are highlighted and the optimum load is plotted with an asterisk. This shows that, with only 7 measurements, a large area of the Smith Chart can be captured and the maximum model order identified for each area. To verify this result for normal $\mathrm{CW}$ measurements, the trace icons in fig. 7 were used as $\mathrm{CW}$ impedance targets and model analysis was performed on the received data.

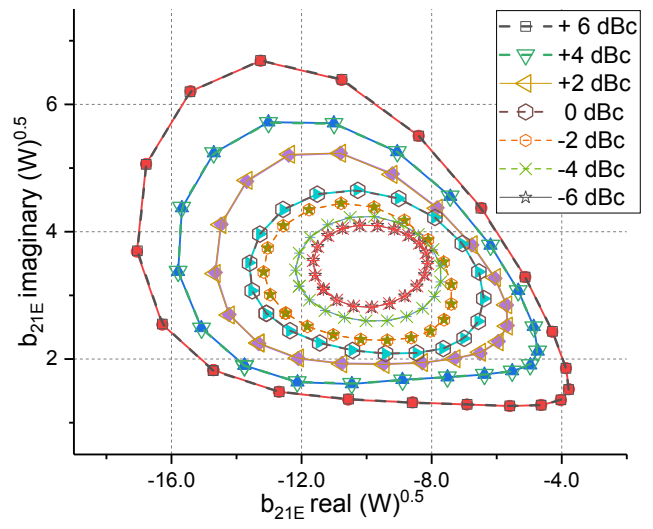

Fig. 6. $b_{21 E}$ modeled and measured comparisons for different $\left|a_{21,2}\right|$ power levels with phase variation only.

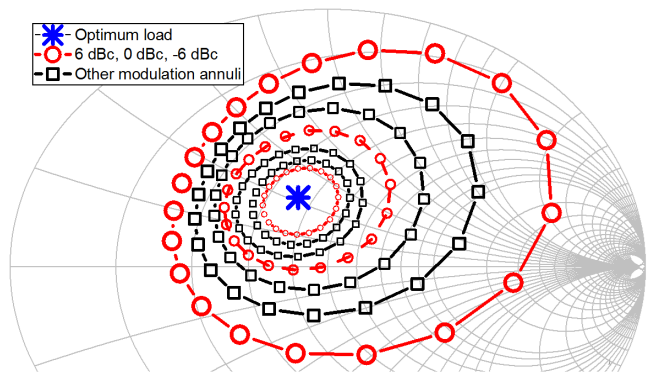

Fig. 7. Load-modulation annuli for different $\left|a_{21,2}\right|$ power levels. Trace icons indicate impedance targets for $\mathrm{CW}$ measurement.

Fig. 8, plots the NMSE, for selected $\left|a_{21,2}\right|$ power levels, against model order for the two-tone and $\mathrm{CW}$ cases. It can be seen that the addition of model coefficients increases the accuracy of the model and that the two-tone and $\mathrm{CW}$ results are offset for $\left|a_{21,2}\right|=0 \mathrm{dBc}$ and $\left|a_{21,2}\right|=6 \mathrm{dBc}$, this is due to an $a_{21}$ that exhibits magnitude and phase variation for the larger annuli in fig. 7. However, it can be seen that the maximum model order, obtained from the two-tone measurements, holds for the CW case (NMSE $<-40 \mathrm{dBm}$ ). In both cases, small gains in accuracy can be achieved after the identified model order, however, the models would be overdetermined.

\section{CONCLUSION}

A new technique, exploiting two-tone measurements, for the correct determination of Cardiff Model phase coefficients has been introduced. Using this technique, the analytical Xparameter model formulation has been shown to remain accurate until $\left|a_{21,2}\right|=2 \mathrm{dBc}$, however, higher order formulations 


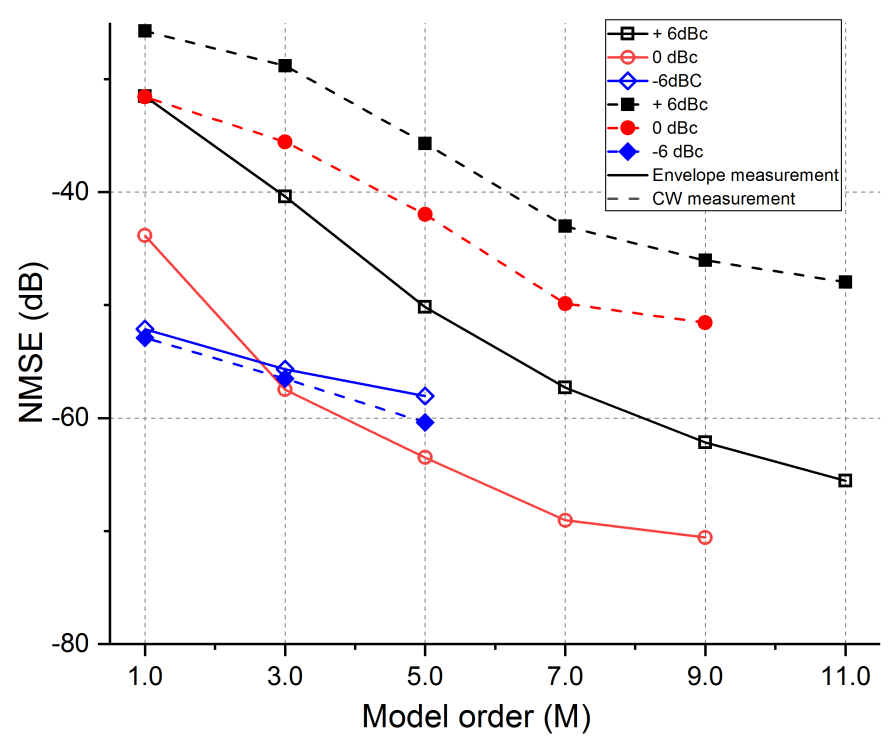

Fig. 8. NMSE versus model order for two-tone measurements at selected $\left|a_{21,2}\right|$ and $\mathrm{CW}$ measurements with impedances lying on their respective two-tone load-modulation annuli.

are required for better accuracy with increasing $\left|a_{21,2}\right|$ power levels. The $\left|a_{21,2}\right|$ perturbation has been increased to cover a substantial area of the Smith Chart and is accurately modeled by an $11^{\text {th }}$ order phase polynomial. This approach allows for the maximum model complexity to be clearly identified directly from measurements, hence allowing for the direct extraction of both the maximum model order as well as the associated coefficients. Using impedance targets obtained from the two-tone measurements, model analysis was performed for $\mathrm{CW}$ measurements over the same area of the Smith Chart, and shows that the model identification can directly be applied to $\mathrm{CW}$ measurements for accurate models that avoid overdetermination.

\section{REFERENCES}

[1] K. Kurokawa, "Power waves and the scattering matrix," vol. 13, no. 2 IEEE, 1965, pp. 194-202.

[2] D. Root et al., "Polyharmonic distortion modeling," IEEE microwave magazine, vol. 7, no. 3, pp. 44-57, 2006.

[3] D. Root, J. Horn, L. Betts, C. Gillease, and J. Verspecht, "X-parameters: The new paradigm for measurement, modeling, and design of nonlinear rf and microwave components," no. 12, 2008, p. 16.

[4] J. Bell, R. Saini, S. Woodington, J. Lees, J. Benedikt, S. Cripps, and P. Tasker, "Behavioral model analysis using simultaneous active fundamental load-pull and harmonic source-pull measurements at x-band," in Microwave Symposium Digest (MTT), 2011 IEEE MTT-S International. IEEE, 2011, pp. 1-4.

[5] P. J. Tasker, "Practical waveform engineering," IEEE Microwave Magazine, vol. 10, no. 7, 2009.

[6] T. Husseini, A. Al-Rawachy, J. Benedikt, J. Bel, and P. Tasker, "Automating the accurate extraction and verification of the cardiff model via the direct measurement of load-pull power contours," in 2018 IEEE/MTT-S International Microwave Symposium-IMS. IEEE, 2018, pp. 544-547. 